\title{
Optimized observable readout from single-shot images of ultracold atoms via machine learning
}

\author{
Axel U. J. Lode $\odot,{ }^{1, *}$ Rui Lin $\odot,{ }^{2}$ Miriam Büttner, ${ }^{1}$ Luca Papariello $\odot,{ }^{3}$ Camille Lévêque,,${ }^{4,5}$ R. Chitra, ${ }^{2}$ Marios C. Tsatsos, ${ }^{6}$ \\ Dieter Jaksch, ${ }^{7,8}$ and Paolo Molignini ${ }^{7,9, \dagger}$ \\ ${ }^{1}$ Institute of Physics, Albert-Ludwig University of Freiburg, Hermann-Herder-Strasse 3, 79104 Freiburg, Germany \\ ${ }^{2}$ Institute for Theoretical Physics, ETH Zürich, 8093 Zurich, Switzerland \\ ${ }^{3}$ Research Studio Data Science, RSA FG, Thurngasse 8/16, 1090 Wien, Austria \\ ${ }^{4}$ Vienna Center for Quantum Science and Technology, Atominstitut, TU Wien, Stadionallee 2, 1020 Vienna, Austria \\ ${ }^{5}$ Wolfgang Pauli Institute c/o Faculty of Mathematics, University of Vienna, Oskar-Morgenstern Platz 1, 1090 Vienna, Austria \\ ${ }^{6}$ Honest AI Ltd., 65 Goswell Road, London ECIV 7EN, United Kingdom \\ ${ }^{7}$ Clarendon Laboratory, University of Oxford, Oxford OX1 3PU, United Kingdom \\ ${ }^{8}$ Institut für Laserphysik, Universität Hamburg, 22761 Hamburg, Germany \\ ${ }^{9}$ Cavendish Laboratory, 19 JJ Thomson Ave, Cambridge CB3 OHE, United Kingdom
}

(Received 20 July 2021; revised 8 September 2021; accepted 8 September 2021; published 8 October 2021)

\begin{abstract}
Single-shot images are the standard readout of experiments with ultracold atoms, the imperfect reflection of their many-body physics. The efficient extraction of observables from single-shot images is thus crucial. Here we demonstrate how artificial neural networks can optimize this extraction. In contrast to standard averaging approaches, machine learning allows both one- and two-particle densities to be accurately obtained from a drastically reduced number of single-shot images. Quantum fluctuations and correlations are directly harnessed to obtain physical observables for bosons in a tilted double-well potential at an extreme accuracy. Strikingly, machine learning also enables a reliable extraction of momentum-space observables from real-space single-shot images and vice versa. With this technique, the reconfiguration of the experimental setup between in situ and time-of-flight imaging is required only once to obtain training data, thus potentially granting an outstanding reduction in resources.
\end{abstract}

DOI: 10.1103/PhysRevA.104.L041301

Ultracold atoms are used as remarkably flexible analog quantum simulators of otherwise hardly accessible quantum many-body states [1-6]. Recent highlights include the quantum simulation of correlations in Fermi-Hubbard systems [7] and the quantization of conductance through a quantum point contact $[8,9]$. An important aspect underpinning all cold-atom-based quantum simulators is that their readout is single-shot images. Experimental techniques for recording single-shot images include photoluminescence or indirect microscopy and tomography [10-14]. These readouts of ultracold atomic systems are projective measurements of the many-body wave function [15-21]. Ideally, such single-shot images (random samples of the $N$-body density) contain information about the position or momentum of every imaged atom and thus about densities and correlation functions to all orders [22-24], quantum fluctuations [25,26], and full distribution functions [27].

A key goal is to have a quantum simulator whose readouts directly furnish information about complex correlation functions and distributions relevant, for instance, to our understanding of the nature of strongly correlated phases. Typically sought observables, one- and two-body correlation functions, are extracted by averaging a sufficiently large number of

\footnotetext{
*auj.lode@gmail.com

†phys2088@ox.ac.uk
}

single-shot images. This averaging corresponds, formally, to the extraction of a marginal distribution via a trace operation. Such a trace operation might not preserve all the information about correlation functions. Reaching towards the goal to optimally readout quantum simulators, we propose to use artificial neural networks (ANNs) to optimally extract a wide range of pertinent observables from single-shot images.

The booming field of machine learning (ML) has led to many notable results concerning the classification of phases of matter with simulated [28-43] or experimental [44-46] data. Artificial neural networks were used to obtain neural-network quantum states, a compressed yet accurate representation of many-body wave functions [47-59], and to devise efficient quantum state tomography and observable extraction in qubit systems [46,60-62]. Applications of ANNs to single-shot images of ultracold atoms so far have focused on classifying phases of the doped Hubbard model [44], detection of phase transitions of the Haldane and Bose-Hubbard models [45], and denoising for single-exposure imaging [63]. A demonstration that ANNs can be applied for the extraction of general observables including correlation functions from single-shot images of ultracold atoms has been lacking.

In this Letter we consider the extraction of observables from projective quantum measurements of many-body states of ultracold atoms as a regression task. We then devise, train, and apply ANNs to it (Fig. 1). Our ANN-based regression yields observables with far superior accuracy as compared 


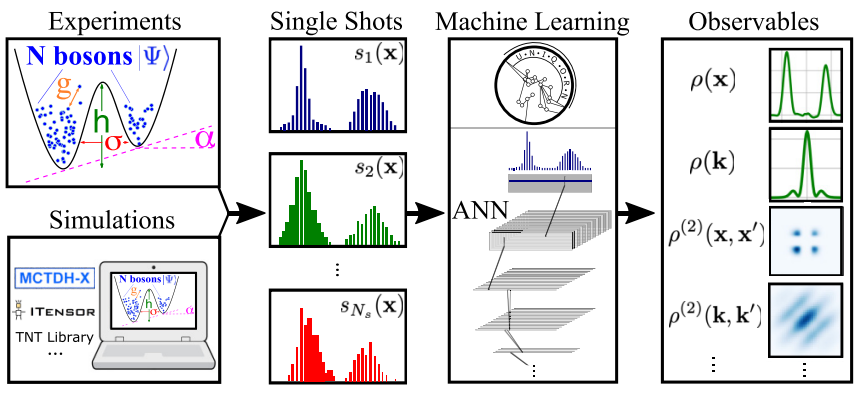

FIG. 1. Machine learning observables from single-shot images of ultracold atomic systems. Single-shot images are measured experimentally or simulated numerically using MCTDH-X or other algorithms and used as input data in an ML task. Other options for wave-function-based approaches that allow the simulation of single-shot measurements include ITensor [64], the TENSOR NETWORK THEORY (label TNT) library [65], or the openMPS software [66,67]. The ANNs are trained on the simulated single-shot data set obtained here for an $N$-boson tilted double-well system to output multiple observables. The quantities $(g, h, \sigma, \alpha, N)$ characterizing this double well are randomized.

to the standard averaging procedure applied to this regression task. As a key result of this Letter, we demonstrate an ANN-based reconstruction of observables from singleshot measurements that would traditionally require a change in the imaging setup: Momentum-space observables can be found from real-space single-shot data and vice versa. The ANN in use has a few convolutional and dense hidden layers and is described in detail in Sec. S1 of the Supplemental Material [68]. The construction, training, and evaluation of ANN models is implemented using TENSORFLOW $[89,90]$ and integrated into a flexible open-source toolkit: the Universal Neural-network Interface for Quantum Observable Readout from $N$-body wavefunctions (UNIQORN) [91].

We aim to demonstrate the potential of our ML algorithm on a physical system that consists of $N$ bosons in a one-dimensional tilted double-well potential described by the Hamiltonian

$$
\begin{aligned}
\mathcal{H}= & \int d \mathbf{x} \hat{\Psi}^{\dagger}(\mathbf{x})[T(\mathbf{x})+V(\mathbf{x})] \hat{\Psi}(\mathbf{x}) \\
& +\frac{1}{2} \int d \mathbf{x} d \mathbf{x}^{\prime} \hat{\Psi}^{\dagger}(\mathbf{x}) \hat{\Psi}^{\dagger}\left(\mathbf{x}^{\prime}\right) W\left(\mathbf{x}, \mathbf{x}^{\prime}\right) \hat{\Psi}\left(\mathbf{x}^{\prime}\right) \hat{\Psi}(\mathbf{x}),
\end{aligned}
$$

where $\hat{\Psi}(\mathbf{x})$ and $\hat{\Psi}^{\dagger}(\mathbf{x})$ are bosonic field operators. The one-body part of the Hamiltonian contains a kinetic energy term $T(\mathbf{x})=-\frac{1}{2} \partial_{x}^{2}$ and a tilted one-body double-well potential $V(\mathbf{x})=\frac{1}{2} \mathbf{x}^{2}+h \exp \left(-\frac{\mathbf{x}^{2}}{\sigma^{2}}\right)+\alpha \mathbf{x}$ that is modeled as a combination of an external harmonic confinement, a central Gaussian barrier of height $h$ and width $\sigma$, and a tilt of slope $\alpha$. The particles interact via a two-body contact repulsion $W\left(\mathbf{x}, \mathbf{x}^{\prime}\right)=g \delta\left(\mathbf{x}-\mathbf{x}^{\prime}\right), g>0$ (Fig. 1). All units are given in terms of the natural length scale $L=\sqrt{\hbar / m \omega}$ and energy scale $E=\hbar \omega$, where $m$ is the mass of the particles and $\omega$ is the external harmonic trapping frequency.

The tilted double-well system can be viewed as a minimal implementation of a quantum simulator of the two-site Hubbard model [7,92], when the shape of the state within each well is ignored. The two lattice sites are then given by the two minima of the one-body potential, the hopping strength is controlled by the barrier height, and the on-site interaction and energy offset are derived from the interaction strength and the tilt, respectively. Despite their apparent simplicity, double wells feature a wealth of many-body properties, e.g., correlations [23,93,94], number fluctuations [95], self-trapping, and equilibration dynamics [96], and are of contemporary experimental interest [22,23,97,98]. Therefore, double wells provide the perfect arena to implement and benchmark our ANN approach to extract observables.

As the data set [99] for our ML tasks, we compute 3000 ground-state wave functions of the double-well system described above. Each individual wave function corresponds to a double-well system whose system parameters barrier height $h \in[5,25]$, barrier width $\sigma \in[0,3]$, interactions $g \in[0.01,0.2]$, tilt $\alpha \in[0,0.5]$, and particle number $N \in$ $[10,100]$ are generated randomly according to a uniform distribution within the corresponding intervals. The ranges for the random parameters are tuned such that the obtained ground states span a range of physical phenomena that are expected for interacting ultracold bosons in double-well potentials. Our data set thus contains wave functions of both condensed and fragmented systems where the reduced onebody density matrix has a single [100] or several $[101,102]$ macroscopic $[O(N)]$ eigenvalues, respectively.

For each wave function $\Psi\left(\mathbf{x}_{1}, \ldots, \mathbf{x}_{N}\right)$ in our data set of solutions of the Schrödinger equation with the Hamiltonian (1), we generate 1000 single-shot images as the input data for our ML tasks. These simulated single-shot images are random samples $\left(\tilde{\mathbf{x}}_{1}, \ldots, \tilde{\mathbf{x}}_{N}\right)$ that are drawn from the $N$-particle density $P\left(\mathbf{x}_{1}, \ldots, \mathbf{x}_{N}\right)=\left|\Psi\left(\mathbf{x}_{1}, \ldots, \mathbf{x}_{N}\right)\right|^{2}$ (see Refs. [19,103]). As the labels for our supervised ML regression tasks, we compute one-body densities (1BDs) and two-body densities (2BDs) that we want to infer from the single-shot input data. These input data and their labels are computed in real and in momentum space and form our labeled data set [99]. For convenience, we discuss the real-space data in the following; the discussion is identical for the momentum-space case, replacing $\mathbf{x} \rightarrow \mathbf{k}$. Since we have a range of different particle numbers, the shot-to-shot fluctuations in the single-shot images in our data set stem from both the finite particle number and quantum fluctuations [27,104]. Hence, individual singleshot images in our data set may be very different from their density labels. This deviation of single-shot images from the density is particularly pronounced for small particle numbers and/or strong quantum correlations [19,103].

To solve the $N$-boson Schrödinger equation for the ground state and to simulate the system's detection in single-shot images [25-27,103,104], we use the multiconfigurational time-dependent Hartree method for indistinguishable particles (MCTDH-X) [26,105-111]. The MCTDH-X employs a fully optimized basis set to obtain an accurate representation of the many-body state (Sec. S2 in [68]). Generally, the MCTDH-X can capture the physics of the system beyond the Hubbard model [112-116].

Inspired by Ref. [7], we study here the ANN-based extraction of 1BDs and 2BDs from single-shot images due to their simplicity and importance. Notably, the 2BD has an important role as the simplest indicator of correlation effects 
(a) $\rho(\mathbf{x})$

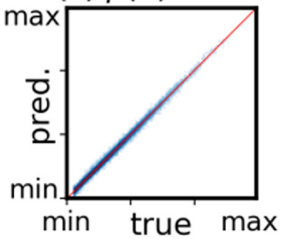

(f) $\rho^{(2)}\left(\mathbf{x}, \mathbf{x}^{\prime}\right)$

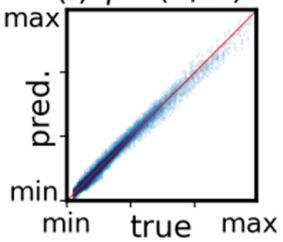

(b)

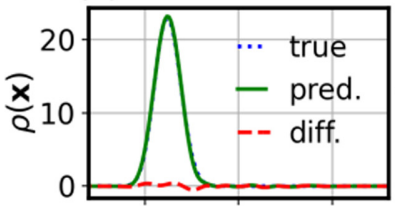

(g)

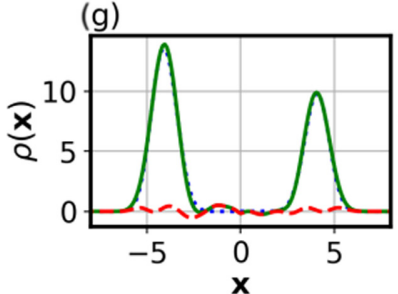

(c) $\rho^{(2)}\left(\mathbf{x}, \mathbf{x}^{\prime}\right)$

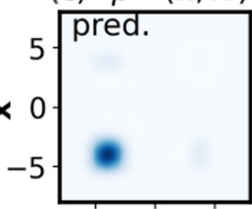

(h) $\rho^{(2)}\left(\mathbf{x}, \mathbf{x}^{\prime}\right)$

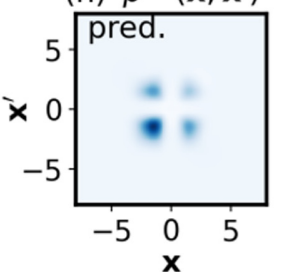

(d) $\rho^{(2)}\left(\mathbf{x}, \mathbf{x}^{\prime}\right)$

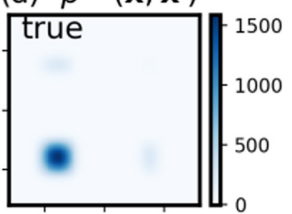

(i) $\rho^{(2)}\left(\mathbf{x}, \mathbf{x}^{\prime}\right)$

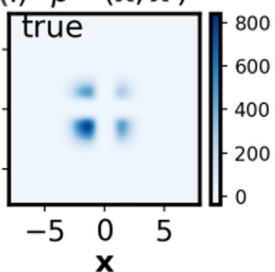

(e)

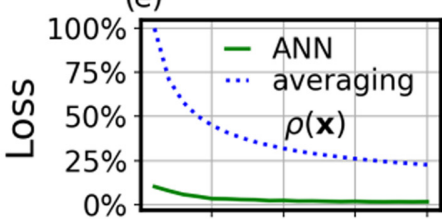

(j)

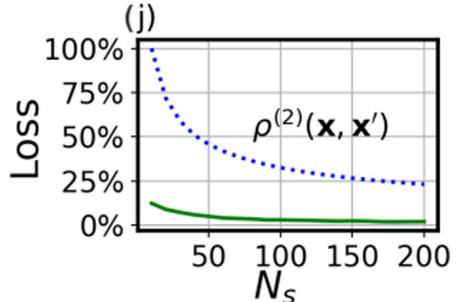

FIG. 2. Regression of real-space (a), (b), (e), and (g) 1BD $\rho(\mathbf{x})$ and (c), (d), (f), and (h)-(j) 2BD $\rho^{(2)}\left(\mathbf{x}, \mathbf{x}^{\prime}\right)$ from real-space single-shot images. (a)-(d) and (f)-(i) Comparisons between predicted (labeled "pred.") and true (labeled "true") values from ANNs with $N_{s}=200$ for (a), (b), and (g) $\rho$ (x) and (c), (d), (f), (h), and (i) $\rho^{(2)}\left(\mathbf{x}, \mathbf{x}^{\prime}\right)$. Each dot in (a) and (f) compares each regressed data point in all test set observables with its corresponding true value. The red line along the diagonal indicates perfect agreement for all ranges. In (b)-(d) one specific condensed state is shown, while in (g)-(i) one specific fragmented state is shown. (e) and (j) Loss function measuring deviation of predictions from true values (Sec. S1 in [68]) as a function of $N_{s}$ compared between the ANN approach and the averaging approach for (e) $\rho(\mathbf{x})$ and (j) $\rho^{(2)}\left(\mathbf{x}, \mathbf{x}^{\prime}\right)$ ( $100 \%$ corresponds to the loss obtained for the averaging formula at $\left.N_{s}=10\right)$. All plotted quantities are dimensionless.

that underpins many-body phenomena beyond mean-field approaches $[93,94]$. We remark that our ANN-based approach works also for other observables like the particle number and the one-body reduced density matrix (Secs. S3 and S4 in [68], respectively).

From the wave function $|\Psi\rangle$, the $1 \mathrm{BD}$ and $2 \mathrm{BD}$ can be evaluated, respectively, as

$$
\begin{gathered}
\rho(\mathbf{x})=\left\langle\Psi\left|\hat{\Psi}^{\dagger}(\mathbf{x}) \hat{\Psi}(\mathbf{x})\right| \Psi\right\rangle / N \\
\rho^{(2)}\left(\mathbf{x}, \mathbf{x}^{\prime}\right)=\left\langle\Psi\left|\hat{\Psi}^{\dagger}(\mathbf{x}) \hat{\Psi}^{\dagger}\left(\mathbf{x}^{\prime}\right) \hat{\Psi}\left(\mathbf{x}^{\prime}\right) \hat{\Psi}(\mathbf{x})\right| \Psi\right\rangle .
\end{gathered}
$$

Figure 2 shows some results of our ANN-based extraction of the 1BD [Eq. (2)] and 2BD [Eq. (3)] in Figs. 2(a)-2(e) and $2(\mathrm{f})-2(\mathrm{j})$, respectively, from a set of simulated real-space single-shot images $\left\{s_{i}(\mathbf{x}), i=1, \ldots, N_{s}\right\}$ as a function of the number of images per input sample $N_{s}$. The detailed description of the ANNs, including the regularizations for preventing overfitting, is shown in Sec. S1 in [68]. In comparison and in contrast to the ANN method, the 1BD and 2BD can also be approximately reconstructed by the averaging approach

$$
\begin{gathered}
\rho(\mathbf{x}) \approx \frac{1}{N_{s}} \sum_{i=1}^{N_{s}} s_{i}(\mathbf{x}), \\
\rho^{(2)}\left(\mathbf{x}, \mathbf{x}^{\prime}\right) \approx \frac{1}{N_{s}} \sum_{i=1}^{N_{s}} s_{i}(\mathbf{x})\left[s_{i}\left(\mathbf{x}^{\prime}\right)-\delta\left(\mathbf{x}, \mathbf{x}^{\prime}\right)\right],
\end{gathered}
$$

where $s_{i}(\mathbf{x})$ are the single-shot images.

In Fig. 2 we illustrate the performance of the ANN-based approach with direct comparisons of the predicted and true values of $\rho$ and $\rho^{(2)}$. Indeed, very good agreement is seen between the predictions and the true values on the full test set [Figs. 2(a) and 2(f)], spanning all physical regimes with very small (bottom left corner) to very large densities (upper right corner). ${ }^{1}$ Furthermore, the predicted densities faithfully reproduce the corresponding true values for all different patterns of coherence. This is exemplified for a condensed [Figs. 2(b)2(d)] and a fragmented [Figs. 2(g) and 2(h)] state. In the fragmented state, spatial correlations between the particles in distinct wells are present: $\rho^{(2)}\left(\mathbf{x}, \mathbf{x}^{\prime}\right)$ is significant at $\left(\mathbf{x}, \mathbf{x}^{\prime}\right)$ where $\mathbf{x}$ lies in one well and $\mathbf{x}^{\prime}$ lies in the other. For the condensed state, such correlations are absent.

To contextualize the performance of the observable reconstruction more concretely, we further explicitly compare the ANN-based accuracy by evaluating the loss function for its predictions on test data. Namely, we directly compare the discrepancy in the ANN-based approach with its counterpart for the averaging approach in Figs. 2(e) and 2(j). Strikingly, the ANN-based approach with $N_{s}=10$ outperforms by more than one order of magnitude the averaging approach for all numbers of single-shot images per sample. This means that the ANN not only drastically reduces the number of singleshot images needed to achieve a certain accuracy, but even grants a better performance with that small number (e.g., $N_{s}=10$ ) when compared to the averaging approach with a large number, i.e., $N_{s}=200$. Even larger numbers of samples are used in some experiments, for instance, $N_{s} \sim O\left(10^{3}\right)$ in the experiments in Refs. [7,23] on extracting correlations for ultracold atoms in double-well systems. We infer that the ANN-based approach can harness the information about the state of the system $|\Psi\rangle$ embedded in the shot-to-shot fluctuations and needed for the construction of observables that bear correlation effects much more efficiently. We remark that the regression of momentum-space $1 \mathrm{BD}$ and $2 \mathrm{BD}$ from

\footnotetext{
${ }^{1}$ Note that the larger values rarefy simply because they typically correspond to states that are completely localized in one side of the double well and are statistically less likely to appear for a randomly distributed range of parameters.
} 
(a) $\rho$ (k)

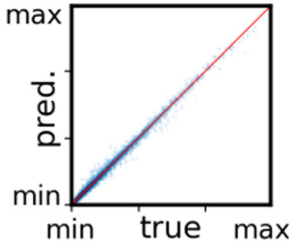

(f) $\rho^{(2)}\left(\mathbf{k}, \mathbf{k}^{\prime}\right)$

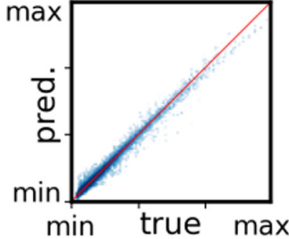

(b)

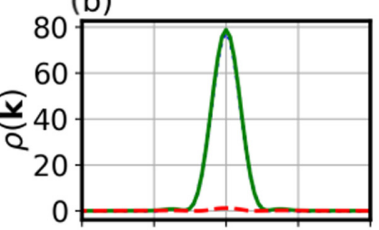

(g)

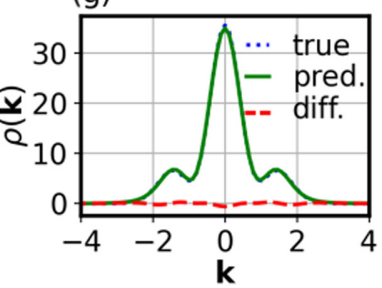

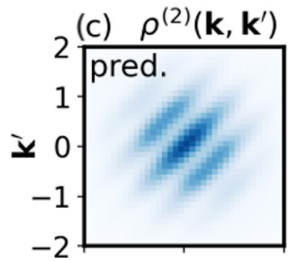

(d) $\rho^{(2)}\left(\mathbf{k}, \mathbf{k}^{\prime}\right)$
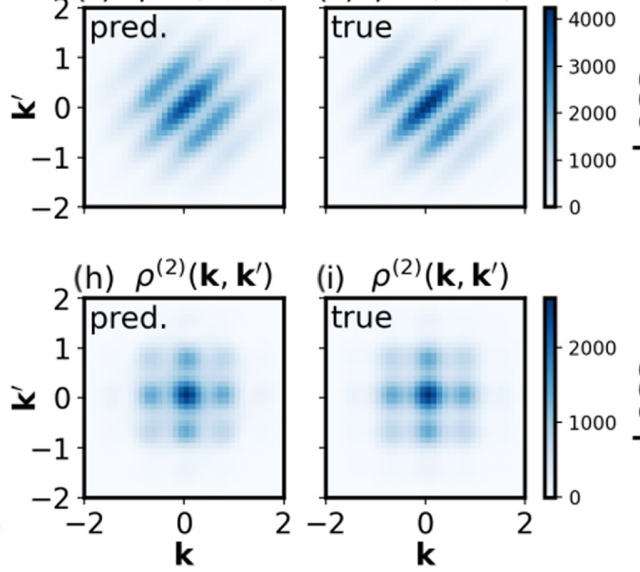

(e)

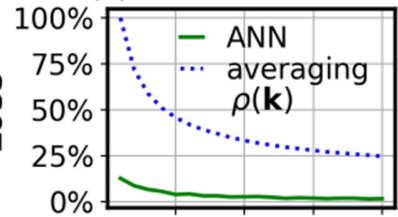

(j)

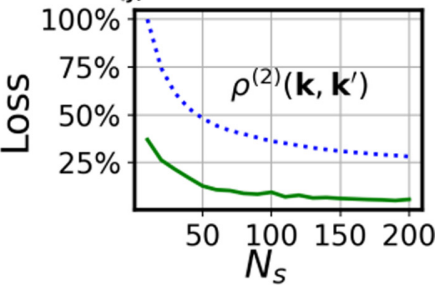

FIG. 3. Regression of momentum-space (a), (b), (e), and (g) 1BD $\rho(\mathbf{k})$ and (c), (d), (f), and (h)-(j) $2 \mathrm{BD} \rho^{(2)}\left(\mathbf{k}, \mathbf{k}^{\prime}\right)$ from real-space single-shot images. (a)-(d), (f), and (i) Comparisons between predicted (labeled "pred.") and true (labeled "true") values from ANNs with $N_{s}=200$ for (a), (b), and (g) $\rho(\mathbf{k})$ and (c), (d), (f), (h), and (i) $\rho^{(2)}\left(\mathbf{k}, \mathbf{k}^{\prime}\right)$. In (a) and (f) the results from the complete test set are shown, in (b)-(d) one specific condensed state is shown, and in (g)-(i) one specific fragmented state is shown. (e) and (j) Loss function measuring deviation of predictions from true values (Sec. S1 in [68]) as a function of $N_{s}$, compared between the ANN and averaging approach for (e) $\rho(\mathbf{k})$ and (j) $\rho^{(2)}\left(\mathbf{k}, \mathbf{k}^{\prime}\right)\left(100 \%\right.$ corresponds to the loss obtained for the averaging formula at $\left.N_{s}=10\right)$. See Sec. S5 in [68] for complementary results on extracting real-space observables from momentum-space single shots $\left\{s_{i}(\mathbf{k}), i=1, \ldots, N_{s}\right\}$. All plotted quantities are dimensionless.

momentum-space single-shot images is performed equally well with the ANN-based approach (see Sec. S6 and Fig. $\mathrm{S} 8$ in [68]). To underpin the generality and relevance of our findings also for experimental data, we demonstrate in Sec. S7 in [68] that the ANN-based approach is remarkably stable against different types of noise. Moreover, we demonstrate in a cross-validation test in Sec. S8 in [68] that an ANN trained on the double-well data set [99] performs similarly well on the prediction of observables for a different data set which we computed for a triple-well setup [117].

Motivated by these promising results for the cases where a benchmark with an averaging formula exists, we now apply ANNs to extract observables for which there exists no method of inference. For this purpose, we consider the problem of inferring momentum-space observables from real-space single-shot images. We thus feed sets of simulated real-space single-shot images $\left\{s_{i}(\mathbf{x})\right\}$ to our ANNs as input data and train them to reconstruct the momentum-space densities $\rho(\mathbf{k})$ and $\rho^{(2)}\left(\mathbf{k}, \mathbf{k}^{\prime}\right)$ [replacing $\mathbf{x} \rightarrow \mathbf{k}$ in Eqs. (2) and (3), respectively].

A conventional formula in the spirit of Eqs. (4) and (5) is unavailable for the inference of momentum-space observables from real-space single-shot images. Nevertheless, we can reconstruct them from momentum-space single-shot images $\left\{s_{i}(\mathbf{k}), i=1, \ldots, N_{s}\right\}$ using Eqs. (4) and (5) after replacing $\mathbf{x} \rightarrow \mathbf{k}$. The loss function of this extraction approach can be used for comparison with the ANN loss function.

Figure 3 shows the performance of the ANN-based extraction of momentum-space observables $\rho(\mathbf{k})$ and $\rho^{(2)}\left(\mathbf{k}, \mathbf{k}^{\prime}\right)$ from real-space single-shot images $\left\{s_{i}(\mathbf{x})\right\}$. Again, the ANNbased extraction yields an outstanding accuracy. The loss function for $N_{s} \approx 200$ is drastically reduced with the ANN approach as compared to the averaging. Additionally, the ANN-based loss function shows an equally good performance at low $N_{s} \approx 10$ as the conventional extraction approach at high $N_{s} \approx 200$. Visually, we again find that all salient features of the $1 \mathrm{BD}$ and $2 \mathrm{BD}$ in momentum space are reproduced with large quantitative accuracy [Figs. 3(b) and 3(g) and Figs. 3(c), 3(d), 3(h), and 3(i), respectively]. Notably, the 2BDs are clearly similar to the fermionic results presented in Ref. [7], confirming again the presence (absence) of correlations in a fragmented (condensed) state. The high accuracy of the ANNbased regression, even when compared to a direct averaging from momentum-space data, suggests that data obtained from in situ imaging setup and processed with ANN architectures could be sufficient to extract reliable momentum-space observables. The potentially time-consuming reconfiguration of the imaging setup to a time-of-flight configuration would be needed only once for obtaining the training data. Therefore, our ANN approach could lead to an extraordinary reduction of laboratory resources.

We thus show that ANN-based observable extraction is a highly promising method for improving the performance of standard measurements in ultracold-atom-based experiments. The impact of our findings is far reaching and multifaceted. Information on a wide range of different states with various degrees of coherence can be retrieved reliably and rapidly. The number of single-shot images and therefore the runtime of a costly experimental setup can be drastically reduced when using an ANN in comparison to when using the conventional averaging approach. Moreover, we demonstrate how to extract momentum-space observables from in situ single-shot images and vice versa without any tedious reconfiguration of the imaging setup and at a very high degree of accuracy. Our results thus herald the potential of ANNs to obtain even more information from ultracold-atom-based quantum simulators.

The next step is to obtain a first proof of concept by applying our ANN-based extraction to experimental singleshot images of single- and double-well setups [7,23,98,118121]. Further applications include the investigation of more involved cold-atom setups beyond single and double wells and tough-to-measure quantities like the potential [122] or 
higher-order densities and correlation functions [24]. Following our promising results of regressing triple-well observables with a neural network trained on double-well data (Sec. S8 and Fig. S11 in [68]), another important future question is to understand which further systems are amenable to regression with the neural network with minimal additional retraining.

This work was supported by the Austrian Science Foundation through Grants No. P-32033-N32 and No. M-2653, the Swiss National Science Foundation and ETH grants, and
EPSRC Grant No. EP/P009565/1 and was partially funded by the European Research Council under the European Union's Seventh Framework Programme/ERC Grant Agreement No. 319286 Q-MAC. Computation time on the Hazel Hen and Hawk clusters at the HLRS Stuttgart and on the ARCUS cluster of the University of Oxford and support by the German Research Foundation and the state of Baden-Württemberg via the bwHPC Grants No. INST 40/467-1 FUGG (JUSTUS cluster), No. INST 39/963-1 FUGG (bwForCluster NEMO), and No. INST 37/935-1 FUGG (bwForCluster BinAC) are gratefully acknowledged.
[1] M. Lewenstein, A. Sanpera, V. Ahufinger, B. Damski, A. Sen(De), and U. Sen, Adv. Phys. 56, 243 (2007).

[2] I. Bloch, J. Dalibard, and W. Zwerger, Rev. Mod. Phys. 80, 885 (2008).

[3] I. Bloch, J. Dalibard, and S. Nascimbène, Nat. Phys. 8, 267 (2012).

[4] I. M. Georgescu, S. Ashhab, and F. Nori, Rev. Mod. Phys. 86, 153 (2014).

[5] C. Gross and I. Bloch, Science 357, 995 (2017).

[6] F. Schäfer, T. Fukuhara, S. Sugawa, Y. Takasu, and Y. Takahashi, Nat. Rev. Phys. 2, 411 (2020).

[7] A. Bergschneider, V. M. Klinkhamer, J. H. Becher, R. Klemt, L. Palm, G. Zürn, S. Jochim, and P. M. Preiss, Nat. Phys. 15, 640 (2019).

[8] S. Krinner, D. Stadler, D. Husmann, J.-P. Brantut, and T. Esslinger, Nature (London) 517, 64 (2015).

[9] L. Corman, P. Fabritius, S. Häusler, J. Mohan, L. H. Dogra, D. Husmann, M. Lebrat, and T. Esslinger, Phys. Rev. A 100, 053605 (2019).

[10] R. Bücker, A. Perrin, S. Manz, T. Betz, C. Koller, T. Plisson, J. Rottmann, T. Schumm, and J. Schmiedmayer, New J. Phys. 11, 103039 (2009).

[11] W. S. Bakr, J. I. Gillen, A. Peng, S. Fölling, and M. Greiner, Nature (London) 462, 74 (2009).

[12] J. F. Sherson, C. Weitenberg, M. Endres, M. Cheneau, I. Bloch, and S. Kuhr, Nature (London) 467, 68 (2010).

[13] D. A. Smith, S. Aigner, S. Hofferberth, M. Gring, M. Andersson, S. Wildermuth, P. Krüger, S. Schneider, T. Schumm, and J. Schmiedmayer, Opt. Express 19, 8471 (2011).

[14] E. Estrecho, T. Gao, N. Bobrovska, M. D. Fraser, M. Steger, L. Pfeiffer, K. West, T. C. H. Liew, M. Matuszewski, D. W. Snoke, A. G. Truscott, and E. A. Ostrovskaya, Nat. Commun. 9, 2944 (2018).

[15] J. Javanainen and S. M. Yoo, Phys. Rev. Lett. 76, 161 (1996).

[16] Y. Castin and J. Dalibard, Phys. Rev. A 55, 4330 (1997).

[17] J. Dziarmaga, Z. P. Karkuszewski, and K. Sacha, J. Phys. B 36, 1217 (2003).

[18] D. Dagnino, N. Barberán, and M. Lewenstein, Phys. Rev. A 80, 053611 (2009).

[19] K. Sakmann and M. Kasevich, Nat. Phys. 12, 451 (2016).

[20] M. Gajda, J. Mostowski, T. Sowiński, and M. Załuska-Kotur, Europhys. Lett. 115, 20012 (2016).

[21] D. Rakshit, J. Mostowski, T. Sowiński, M. Załuska-Kotur, and M. Gajda, Sci. Rep. 7, 15004 (2017).

[22] T. Langen, S. Erne, R. Geiger, B. Rauer, T. Schweigler, M. Kuhnert, W. Rohringer, I. E. Mazets, T. Gasenzer, and J. Schmiedmayer, Science 348, 207 (2015).
[23] T. Schweigler, V. Kasper, S. Erne, I. Mazets, B. Rauer, F. Cataldini, T. Langen, T. Gasenzer, J. Berges, and J. Schmiedmayer, Nature (London) 545, 323 (2017).

[24] C. Lévêque, F. Diorico, J. Schmiedmayer, and A. U. J. Lode, arXiv:2006.10755.

[25] B. Chatterjee and A. U. J. Lode, Phys. Rev. A 98, 053624 (2018).

[26] R. Lin, P. Molignini, L. Papariello, M. C. Tsatsos, C. Lévêque, S. E. Weiner, E. Fasshauer, R. Chitra, and A. U. J. Lode, Quantum Sci. Technol. 5, 024004 (2020).

[27] B. Chatterjee, C. Lévêque, J. Schmiedmayer, and A. U. J. Lode, Phys. Rev. Lett. 125, 093602 (2020).

[28] L. Wang, Phys. Rev. B 94, 195105 (2016).

[29] G. Torlai and R. G. Melko, Phys. Rev. B 94, 165134 (2016).

[30] P. Broecker, J. Carrasquilla, R. G. Melko, and S. Trebst, Sci. Rep. 7, 8823 (2017).

[31] J. Carrasquilla and R. G. Melko, Nat. Phys. 13, 431 (2017).

[32] K. Ch'ng, J. Carrasquilla, R. G. Melko, and E. Khatami, Phys. Rev. X 7, 031038 (2017).

[33] E. P. L. van Nieuwenburg, Y.-H. Liu, and S. D. Huber, Nat. Phys. 13, 435 (2017).

[34] S. J. Wetzel, Phys. Rev. E 96, 022140 (2017).

[35] W. Hu, R. R. P. Singh, and R. T. Scalettar, Phys. Rev. E 95, 062122 (2017).

[36] Y. Zhang and E.-A. Kim, Phys. Rev. Lett. 118, 216401 (2017).

[37] F. Schindler, N. Regnault, and T. Neupert, Phys. Rev. B 95, 245134 (2017).

[38] N. C. Costa, W. Hu, Z. J. Bai, R. T. Scalettar, and R. R. P. Singh, Phys. Rev. B 96, 195138 (2017).

[39] K. Ch'ng, N. Vazquez, and E. Khatami, Phys. Rev. E 97, 013306 (2018).

[40] W.-J. Rao, Z. Li, Q. Zhu, M. Luo, and X. Wan, Phys. Rev. B 97, 094207 (2018).

[41] Z. Li, M. Luo, and X. Wan, Phys. Rev. B 99, 075418 (2019).

[42] M. S. Scheurer and R.-J. Slager, Phys. Rev. Lett. 124, 226401 (2020).

[43] J. Arnold, F. Schäfer, M. Žonda, and A. U. J. Lode, Phys. Rev. Research 3, 033052 (2021).

[44] A. Bohrdt, C. S. Chiu, G. Ji, M. Xu, D. Greif, M. Greiner, E. Demler, F. Grusdt, and M. Knap, Nat. Phys. 15, 921 (2019).

[45] B. S. Rem, N. Käming, M. Tarnowski, L. Asteria, N. Fläschner, C. Becker, K. Sengstock, and C. Weitenberg, Nat. Phys. 15, 917 (2019).

[46] M. Neugebauer, L. Fischer, A. Jäger, S. Czischek, S. Jochim, M. Weidemüller, and M. Gärttner, Phys. Rev. A 102, 042604 (2020).

[47] G. Carleo and M. Troyer, Science 355, 602 (2017). 
[48] X. Gao and L.-M. Duan, Nat. Commun. 8, 662 (2017).

[49] Y. Nomura, A. S. Darmawan, Y. Yamaji, and M. Imada, Phys. Rev. B 96, 205152 (2017).

[50] D.-L. Deng, X. Li, and S. Das Sarma, Phys. Rev. X 7, 021021 (2017).

[51] D.-L. Deng, X. Li, and S. Das Sarma, Phys. Rev. B 96, 195145 (2017).

[52] I. Glasser, N. Pancotti, M. August, I. D. Rodriguez, and J. I. Cirac, Phys. Rev. X 8, 011006 (2018).

[53] G. Torlai, G. Mazzola, J. Carrasquilla, M. Troyer, R. Melko, and G. Carleo, Nat. Phys. 14, 447 (2018).

[54] Z. Cai and J. Liu, Phys. Rev. B 97, 035116 (2018).

[55] J. Chen, S. Cheng, H. Xie, L. Wang, and T. Xiang, Phys. Rev. B 97, 085104 (2018).

[56] R. Kaubruegger, L. Pastori, and J. C. Budich, Phys. Rev. B 97, 195136 (2018).

[57] G. Carleo, Y. Nomura, and M. Imada, Nat. Commun. 9, 5322 (2018).

[58] K. Choo, G. Carleo, N. Regnault, and T. Neupert, Phys. Rev. Lett. 121, 167204 (2018).

[59] T. Vieijra, C. Casert, J. Nys, W. De Neve, J. Haegeman, J. Ryckebusch, and F. Verstraete, Phys. Rev. Lett. 124, 097201 (2020).

[60] G. Torlai, G. Mazzola, G. Carleo, and A. Mezzacapo, Phys. Rev. Research 2, 022060(R) (2020).

[61] G. Torlai, B. Timar, E. P. L. van Nieuwenburg, H. Levine, A. Omran, A. Keesling, H. Bernien, M. Greiner, V. Vuletić, M. D. Lukin, R. G. Melko, and M. Endres, Phys. Rev. Lett. 123, 230504 (2019).

[62] J. Carrasquilla, G. Torlai, R. G. Melko, and L. Aolita, Nat. Mach. Intell. 1, 155 (2019).

[63] G. Ness, A. Vainbaum, C. Shkedrov, Y. Florshaim, and Y. Sagi, Phys. Rev. Appl. 14, 014011 (2020).

[64] M. Fishman, S. R. White, and E. M. Stoudenmire, arXiv:2007.14822.

[65] S. Al-Assam, S. R. Clark, and D. Jaksch, J. Stat. Mech. (2017) 093102.

[66] M. L. Wall and L. D. Carr, New J. Phys. 14, 125015 (2012).

[67] D. Jaschke, M. L. Wall, and L. D. Carr, Comput. Phys. Commun. 225, 59 (2018).

[68] See Supplemental Material at http://link.aps.org/ supplemental/10.1103/PhysRevA.104.L041301 for a discussion of the ML methods used, the MCTDH-X theory, the ANN-based extraction of the particle number, and the reduced one-body density matrix. (References [69-88] are cited therein as well.).

[69] Y. Lecun, L. Bottou, Y. Bengio, and P. Haffner, Proc. IEEE 86, 2278 (1998).

[70] Y. Levine, O. Sharir, N. Cohen, and A. Shashua, Phys. Rev. Lett. 122, 065301 (2019).

[71] A. LeNail, J. Open Source Softw. 4, 747 (2019).

[72] I. Goodfellow, Y. Bengio, and A. Courville, Deep Learning (MIT Press, Cambridge, 2016)

[73] G. Klambauer, T. Unterthiner, A. Mayr, and S. Hochreiter, Adv. Neural Inf. Process. Syst. 30, 6698 (2017).

[74] J. Zanghellini, M. Kitzler, C. Fabian, T. Brabec, and A. Scrinzi, Laser Phys. 13, 1064 (2003).

[75] L. Cao, S. Krönke, O. Vendrell, and P. Schmelcher, J. Chem. Phys. 139, 134103 (2013).
[76] L. Cao, V. Bolsinger, S. I. Mistakidis, G. M. Koutentakis, S. Krönke, J. M. Schurer, and P. Schmelcher, J. Chem. Phys. 147, 044106 (2017).

[77] M. H. Beck, A. Jäckle, G. A. Worth, and H. D. Meyer, Phys. Rep. 324, 1 (2000).

[78] D. J. Haxton and C. W. McCurdy, Phys. Rev. A 91, 012509 (2015).

[79] O. E. Alon, A. I. Streltsov, and L. S. Cederbaum, Phys. Rev. A 76, 062501 (2007).

[80] O. E. Alon, A. I. Streltsov, K. Sakmann, A. U. J. Lode, J. Grond, and L. S. Cederbaum, Chem. Phys. 401, 2 (2012).

[81] H. Miyagi and L. B. Madsen, Phys. Rev. A 87, 062511 (2013).

[82] H. Miyagi and L. B. Madsen, Phys. Rev. A 95, 023415 (2017).

[83] C. Lévêque and L. B. Madsen, New J. Phys. 19, 043007 (2017).

[84] C. Lévêque and L. B. Madsen, J. Phys. B 51, 155302 (2018).

[85] J. S. Smith, B. T. Nebgen, R. Zubatyuk, N. Lubbers, C. Devereux, K. Barros, S. Tretiak, O. Isayev, and A. E. Roitberg, Nat. Commun. 10, 2903 (2019).

[86] R. Zen, L. My, R. Tan, F. Hébert, M. Gattobigio, C. Miniatura, D. Poletti, and S. Bressan, Phys. Rev. E 101, 053301 (2020).

[87] C.-K. Lee, C. Lu, Y. Yu, Q. Sun, C.-Y. Hsieh, S. Zhang, Q. Liu, and L. Shi, J. Chem. Phys. 154, 024906 (2021).

[88] S. Ioffe and C. Szegedy, Proceedings of the 32nd International Conference on Machine Learning, Vol. 37 (PMLR, 2015), pp. 448-456.

[89] M. Abadi et al., TensorFlow: Large-scale machine learning on heterogeneous systems, https://www.tensorflow.org/ (Google Brain, Mountain View, 2015).

[90] F. Chollet et al., Keras, https://keras.io/ (MIT, Cambridge, 2015).

[91] A. U. J. Lode, P. Molignini, R. Lin, M. Büttner, P. Rembold, C. Lévêque, M. C. Tsatsos, and L. Papariello, UNIQORN: Universal neural-network interface for quantum observable readout from $N$-body wavefunctions, https://gitlab. com/UNIQORN/uniqorn (2021).

[92] S. Murmann, A. Bergschneider, V. M. Klinkhamer, G. Zürn, T. Lompe, and S. Jochim, Phys. Rev. Lett. 114, 080402 (2015).

[93] K. Sakmann, A. I. Streltsov, O. E. Alon, and L. S. Cederbaum, Phys. Rev. A 78, 023615 (2008).

[94] K. Sakmann, A. I. Streltsov, O. E. Alon, and L. S. Cederbaum, Phys. Rev. A 89, 023602 (2014).

[95] K. Sakmann, A. I. Streltsov, O. E. Alon, and L. S. Cederbaum, Phys. Rev. A 84, 053622 (2011)

[96] K. Sakmann, A. I. Streltsov, O. E. Alon, and L. S. Cederbaum, Phys. Rev. Lett. 103, 220601 (2009).

[97] R. Bücker, J. Grond, S. Manz, T. Berrada, T. Betz, C. Koller, U. Hohenester, T. Schumm, A. Perrin, and J. Schmiedmayer, Nat. Phys. 7, 608 (2011).

[98] M. Pigneur, T. Berrada, M. Bonneau, T. Schumm, E. Demler, and J. Schmiedmayer, Phys. Rev. Lett. 120, 173601 (2018).

[99] A. U. J. Lode, R. Lin, M. Büttner, P. Rembold, C. Lévêque, M. C. Tsatsos, P. Molignini, and L. Papariello, UNIQORN data set, https://drive.google.com/file/d/ 1y1cZWaAo57e4CVL_jv9Q6T3E6Q4XHh-I/view?usp= sharing (2021).

[100] O. Penrose and L. Onsager, Phys. Rev. 104, 576 (1956).

[101] R. W. Spekkens and J. E. Sipe, Phys. Rev. A 59, 3868 (1999).

[102] P. Nozieres and D. St. James, J. Phys. (Paris) 43, 1133 (1982). 
[103] A. U. J. Lode and C. Bruder, Phys. Rev. Lett. 118, 013603 (2017).

[104] J. H. V. Nguyen, M. C. Tsatsos, D. Luo, A. U. J. Lode, G. D. Telles, V. S. Bagnato, and R. G. Hulet, Phys. Rev. X 9, 011052 (2019).

[105] O. E. Alon, A. I. Streltsov, and L. S. Cederbaum, J. Chem. Phys. 127, 154103 (2007).

[106] A. I. Streltsov, O. E. Alon, and L. S. Cederbaum, Phys. Rev. Lett. 99, 030402 (2007).

[107] O. E. Alon, A. I. Streltsov, and L. S. Cederbaum, Phys. Rev. A 77, 033613 (2008)

[108] A. U. J. Lode, Phys. Rev. A 93, 063601 (2016).

[109] E. Fasshauer and A. U. J. Lode, Phys. Rev. A 93, 033635 (2016).

[110] A. U. J. Lode, M. C. Tsatsos, E. Fasshauer, R. Lin, L. Papariello, P. Molignini, S. E. Weiner, and C. Lévêque, MCTDH-X: The multiconfigurational time-dependent Hartree for indistinguishable particles software, http://ultracold.org (MCTDH-X software, 2020).

[111] A. U. J. Lode, C. Lévêque, L. B. Madsen, A. I. Streltsov, and O. E. Alon, Rev. Mod. Phys. 92, 011001 (2020).

[112] S. I. Mistakidis, L. Cao, and P. Schmelcher, J. Phys. B 47, 225303 (2014).
[113] S. I. Mistakidis, L. Cao, and P. Schmelcher, Phys. Rev. A 91, 033611 (2015).

[114] J. Neuhaus-Steinmetz, S. I. Mistakidis, and P. Schmelcher, Phys. Rev. A 95, 053610 (2017).

[115] S. I. Mistakidis and P. Schmelcher, Phys. Rev. A 95, 013625 (2017).

[116] S. Dutta, M. C. Tsatsos, S. Basu, and A. U. J. Lode, New J. Phys. 21, 053044 (2019).

[117] A. U. J. Lode, R. Lin, M. Büttner, P. Rembold, C. Lévêque, M. C. Tsatsos, P. Molignini, and L. Papariello, UNIQORN triple-well data set, https://drive.google.com/file/d/ 1Zqc8wyzeqWrna-7uMJ9XI_WFreQBJzDu/view?usp= sharing (2021).

[118] M. Albiez, R. Gati, J. Fölling, S. Hunsmann, M. Cristiani, and M. K. Oberthaler, Phys. Rev. Lett. 95, 010402 (2005).

[119] E. Kierig, U. Schnorrberger, A. Schietinger, J. Tomkovic, and M. K. Oberthaler, Phys. Rev. Lett. 100, 190405 (2008).

[120] R. Desbuquois, M. Messer, F. Görg, K. Sandholzer, G. Jotzu, and T. Esslinger, Phys. Rev. A 96, 053602 (2017).

[121] M. Prüfer, T. V. Zache, P. Kunkel, S. Lannig, A. Bonnin, H. Strobel, J. Berges, and M. K. Oberthaler, Nat. Phys. 16, 1012 (2020).

[122] M. Büttner et al. (unpublished). 